\title{
Análisis de caso del modelo de gestión asociativo de la empresa agroindustrial "Don Jorge"
}

\author{
Case Analysis of the Associative Management Model \\ of the Agroindustrial Company "Don Jorge"
}

Análise de caso do modelo de gestão associativo da empresa agroindustrial "Dom Jorge"

\author{
Miguel Ángel Enríquez-Estrella \\ Universidad Estatal Amazónica. Puyo, Ecuador \\ menriquez@uea.edu.ec \\ https://orcid.org/0000-0002-8937-9664
}

\section{Alisson Carolina Reyes Jiménez \\ Universidad Estatal Amazónica. Puyo, Ecuador \\ agi2015045@uea.edu.ec \\ https://orcid.org/0000-0002-6113-3830}

DOI: https://doi.org/10.32719/25506641.2022.11.7

Recibido: 15 de enero de $2021 \cdot$ Revisado: 1 de marzo de 2021

Aceptado: 15 de abril de 2020 • Publicado: 1 de enero de 2022

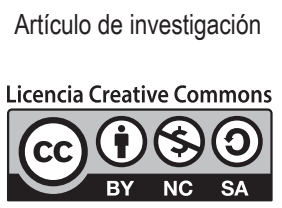




\section{Resumen}

La dinámica de la gestión empresarial ha evolucionado satisfactoriamente en el mundo de los negocios y está imbricada en el mercado, entorno y sociedad, donde se han desarrollado distintas teorías para su aplicación. Esta investigación tiene como objetivo proponer un modelo de asociatividad para la empresa agroindustrial "Don Jorge" que elabora y comercializa té verde en la provincia de Pastaza. La problemática que origina esta investigación es el desconocimiento organizativo y la falta de una estructura que permita el desarrollo socioeconómico del sector con base en el emprendimiento. El método se basa en una investigación bibliográfica, de campo, exploratoria y descriptiva operacionalizando dos variables: asociatividad y mercado. Como resultado se generó un modelo de gestión basado en cuatro ejes fundamentales: estrategia, ejecución, estructura y cultura. El modelo consta de cinco fases: promoción, confianza, plan estratégico y autogestión; con esta estructura la empresa crece e incrementa la confianza, y le permitirá fomentar el desarrollo sustentable de la provincia.

Palabras clave: Organización, estructura, modelo, tecnología, proceso.

JEL: D23 Comportamiento organizativo, costos y derechos de propiedad.

\section{Abstract}

The dynamics of business management has evolved successfully in the world of business and it is embedded in the market, the environment, and society, where different theories have been developed for the application thereof. This research aims to propose a model of associativity for Agroindustrial company "Don Jorge," which produces and sells green tea in the province of Pastaza. The issue leading to this research is the organizational disregard and the lack of a structure that allows the socio-economic growth of the sector based on entrepreneurship. The method relies on bibliographic, field, exploratory, and descriptive research focused on two variables: associativity and market. This led to the creation of a management model based on four fundamental pillars: strategy, execution, structure, and culture. The model consists of five phases: promotion, realibily, strategic planning, and self-management. This structure will help the company grow and increase its reliability, thereby allowing it to promote the sustainable development of the province.

Keywords: Organization, structure, model, technology, process

JEL: D23 Organizational behavior, costs and property rights.

\section{Resumo}

A dinâmica da gestão empresarial tem evolucionado satisfatoriamente no mundo dos negócios e está imbricada no mercado, entorno e sociedade, onde tem-se desenvolvido distintas teorias para sua aplicação. Esta investigação tem o objetivo de propor um modelo de agregação para a empresa Agroindustrial "Dom Jorge" que elabora e comercializa chá verde na província de Pastaza. A problemática que origina esta pesquisa é o desconhecimento organizacional e a falta de uma estrutura que permita o desenvolvimento 
socioeconômico do setor com base no empreendimento. O método fundamenta-se numa investigação bibliográfica, de campo, analítica e descritiva que opera duas variantes: associatividade e mercado. Como resultado gerou-se um modelo de gestão apoiado em quatro eixos fundamentais: estratégia, execução, estrutura e cultura. O modelo tem cinco fases que são a promoção, confiança, plano estratégico e autogestão, com esta estrutura a empresa cresce e incrementa a confiança o que lhe permitirá fomentar o desenvolvimento sustentável da província.

Palavras-chave: Organização, estrutura, modelo, tecnologia, processo JEL: D23 Comportamento organizacional, custos e direitos de propriedade.

\section{Introducción}

L

os sistemas de gestión empresarial se implementan tomando como referencia los sistemas de gestión recomendados por normas internacionales y el estudio analítico del conocimiento acumulado que hace parte de la investigación documental y que tiene como objetivo inventariar y sistematizar la producción en un área del conocimiento, ejercicio que no debe quedarse tan solo en el inventario, sino que debe trascender más allá porque permite hacer una reflexión profunda sobre las tendencias y vacíos en un área específica (Vargas y Calvo 1987).

La mayoría de los países contempla en el comercio internacional el factor que les puede proveer de mayores niveles de crecimiento económico, toda vez que históricamente ha sido superior la tasa a la cual ha crecido el intercambio de bienes y servicios entre las naciones en comparación con el crecimiento de las economías, consideradas individualmente. El comercio mundial, como proporción del PIB mundial, ha evolucionado desde el 1\% que representaba en 1820 , al $6 \%$ en 1900 , luego al $12 \%$ en 1950 , hasta el $26 \%$ para 2000 (BIRF 2009). El enfoque que más se impone es el que considera que la inserción de las economías de América Latina en el comercio internacional es un componente clave en sus respectivas agendas de desarro1lo, puesto que propende por brindarles un crecimiento de calidad, sostenible e incluyente (CAF 2005).

Diversas áreas se han preocupado por generar y difundir conocimientos para ser utilizados por una variedad de organizaciones, entre ellas las empresas familiares. Desde hace poco más de dos décadas. La gestión de empresas 
familiares es una disciplina joven, donde la mayoría de la información generada es prescriptiva y no permite la comparación y generalización de los diferentes estudios. En el caso de Latinoamérica, el estudio de las empresas familiares reviste particular importancia debido a la gran influencia de la familia sobre la empresa, ya que la inmensa mayoría de las empresas están controladas por familias. Según Elizondo (2015), el desarrollo económico del Ecuador se basa en el fortalecimiento de su economía rural a través de la transformación continua, que organiza las fases de distribución, producción, movimiento y consumo de bienes y servicios. Por su parte, Porter (1997) identifica las ventajas competitivas, es decir, aprovechar oportunidades que serán el resultado de la capacidad de la asociación, que les permita ser diferentes de la competencia, la cadena de valor es el análisis de todas las actividades que generen valor agregado y los márgenes que estas produzcan, además de la mejora en la calidad de los productos y en el conocimiento técnico, productivo y comercial, logrando el acceso a nuevos mercados nacionales e internacionales, con una oferta exportable viable.

Las pequeñas y medianas empresas del sector industrial desempeñan un papel muy importante en la economía, principalmente por su contribución en la generación de empleo y por su aporte al proceso de renovación del tejido productivo. La evolución histórica de la gestión empresarial tiene un desenvolvimiento de ideas a nivel cultural en Occidente y Oriente, llegando al desarrollo del hombre en cada uno de los sistemas sociales por los que ha pasado (Fernández 2005), esta ha evolucionado la toma decisiones analizando sus cuatros funciones claves para el desarrollo de mando a nivel empresarial: planificar, organizar, dirigir y controlar. Por tal motivo, la gestión y las personas en el mundo administrativo-empresarial generan grandes competencias.

La empresa agroindustrial "Don Jorge" se dedica a comercializar agua envasada e infusiones de forma rentable; está consciente del rápido crecimiento en cuanto a competitividad en la zona, así como las ventas de sus productos. Es por eso que necesita una estructura empresarial para la mejora continua de sus procesos y ventas. Con el fin de mejorar los servicios hacia los clientes. La trasformación de la materia prima juega un papel interesante en la economía del Ecuador en el sector agrario, sin embargo, la expansión de una empresa o un emprendimiento se hace complicada ya sea por las ofertas o demandas (Enríquez y Pérez 2020). La provincia de Pastaza cuenta 
con el $78 \%$ de la población que consume infusión de hierbas aromáticas, con mayor preferencia el sexo masculino con un $53 \%$; el té es consumido por los atributos y beneficios que posee.

Por la misma razón que representa una estructura oligopolio que excluye a los pequeños emprendedores que están iniciando, según Torres (2016), el modelo asociativo es importante no solo por el impacto económico, sino también por el impacto social ya que constituye la oportunidad de conocer, investigar, observar y percibir oportunidades las cuales van más allá de los límites de la corporación, tomando en cuenta que actualmente es muy escaso este análisis en un país en desarrollo.

La falta de un modelo de gestión asociativo en la empresa "Don Jorge" genera una desventaja de la organización frente a otras que sí la poseen. El proceso de compra y venta hoy en día es un factor importante y determinante para las decisiones de cualquier persona o sociedad, basándose en la calidad del producto y servicio. En la actualidad existe mucha competencia. En el mercado nacional el cliente es un factor importante, ya que es quien impone las condiciones como calidad e innovación inclinándose por lo saludable. La empresa "Don Jorge" comercializa agua embotellada e infusiones que elaboran en sus instalaciones y distribuye en la provincia de Pastaza. Con el objetivo de encontrar oportunidades, en un tiempo corto y que generen mejoras para posicionarse adecuadamente en el mercado local. Se desarrollan habilidades de competitividad en el personal asegurando así la calidad del producto. La Fundación Observatorio PyME (2015) considera que es posible mejorar la productividad mediante un modelo de gestión asociativo que mejore la eficiencia de la empresa y genere un incremento en su economía. Gil Estallo (2007) describe que la empresa es una realidad de nuestra concepción teórica, de nuestro entorno. Se toma en cuenta varios conceptos de empresa: Caldas, Carrión y Heras (2009) la definen como una unidad económica que, a partir de la combinación organizada de diferentes factores materiales y humanos, produce un bien o un servicio destinado a conseguir un beneficio económico; Campiña Domínguez y Fernández Hernández (2010) la definen como el conjunto de actividades llevadas a cabo por el empresario para la producción e intercambio de bienes y servicios con el objeto de obtener un beneficio máximo o por lo menos satisfactorio, y Alegre, Berné Manero y Galve (2008), la definen como la realización de una actividad productiva o 
transformadora; a partir de unos recursos o factores (inputs) obtienen unos productos (outputs, que pueden ser bienes, productos tangibles o servicios, productos intangibles) de mayor valor o utilidad.

La propuesta de la investigación es definir nuevas estructuras productivas y comerciales que eleven el rendimiento productivo y empresarial y buscar ejes estratégicos que mejoren la eficiencia de la empresa.

\section{Marco teórico}

\section{Las ciencias administrativas o de la gestión en la mirada epistemológica}

Cabe destacar que las ciencias administrativas o de la gestión tienen diversos aspectos u obstáculos epistemológicos en la construcción de un nuevo conocimiento, como lo enuncia Contreras Soto (2010) en la tabla 1.

Tabla 1

\section{Obstáculos epistemológicos}

\begin{tabular}{|l|l|}
\hline \multicolumn{2}{|c|}{ Aspectos u obstáculos epistemológicos en la construcción de un nuevo conocimiento } \\
\hline La ausencia y carencia de reflexión teórica en el campo. & $\begin{array}{l}\text { La falta de fundamentos sólidos en la for- } \\
\text { mación disciplinaria. }\end{array}$ \\
\hline $\begin{array}{l}\text { La aplicación de técnicas de investigación bajo crite- } \\
\text { rios de usos y costumbres de la ciencia, sin cuestio- } \\
\text { narse sobre la pertinencia en los objetos de estudios. }\end{array}$ & $\begin{array}{l}\text { La falta de dispositivos orientados a la for- } \\
\text { mación científica en los planes de estudio. }\end{array}$ \\
\hline $\begin{array}{l}\text { Las escalonadas jerarquías de la verdad-autoridad } \\
\text { de la estructura de poder académico, distribuidos pi- } \\
\text { ramidalmente por grados académicos. }\end{array}$ & $\begin{array}{l}\text { Las formulaciones y las acciones del ejerci- } \\
\text { cio de la administración que están atrapadas } \\
\text { en la razón instrumental en la formulación de } \\
\text { la eficiencia. }\end{array}$ \\
\hline $\begin{array}{l}\text { El consumo acrítico de residuos teóricos, principal- } \\
\text { mente del management. }\end{array}$ & $\begin{array}{l}\text { Los problemas complejos de sus objetos } \\
\text { de estudio y de las condiciones sociales } \\
\text { económicas en la periferia. }\end{array}$ \\
\hline
\end{tabular}

Fuente: Contreras Soto (2010). 


\section{Modelo científico funcional}

Las escuelas clásicas de la administración del siglo XX, y sus principales representantes, Frederick W. Taylor, Henry L. Gantt y William Gilbreth, por mencionar algunos, establecieron modelos de administración para las empresas americanas y europeas en las primeras décadas del siglo XX.

La administración de las empresas científicas se centra en la observación de los acontecimientos en la producción, investigación y análisis de las operaciones empresariales, enfocándose en técnicas específicas como son el estudio de tiempo y movimiento, planeación y control en la producción, distribución de equipos en las plantas, reconocimiento salarial, administración del recurso humano, según se detalla en la figura 1.

\section{La administración centrada en la organización y la gestión}

El alumbramiento del tercer milenio planteó al administrador y a la administración la tarea fundamental de comprender la naturaleza de los cambios organizacionales (Torres 2001); señala además dos aspectos que contribuyen a una mejor apreciación de dichas trasformaciones. Los hechos que limitan la posibilidad de renovación de las rutinas organizacionales son dos: la incertidumbre sobre la eficiencia y efectividad de las nuevas formas de organizar los procesos de innovación tecnológica y los altos costos que debe asumir la empresa para cambiar las formas organizativas. La trasformación de las rutinas organizacionales, implica asumir la incertidumbre como un factor que orienta las discusiones y las decisiones de introducir innovaciones en la empresa.

\section{Los modelos y cambios mediáticos}

El concepto de modelo de negocio, que es tan antiguo como la formulación de la teoría de la gestión, resurge en los últimos quince años ante el cambio que supone la transformación de la sociedad de la información y la irrupción del internet. Como en la formulación de la teoría y la práctica de la gestión, es Peter Drucker quien en 1954 habla por primera vez de los modelos de negocio 


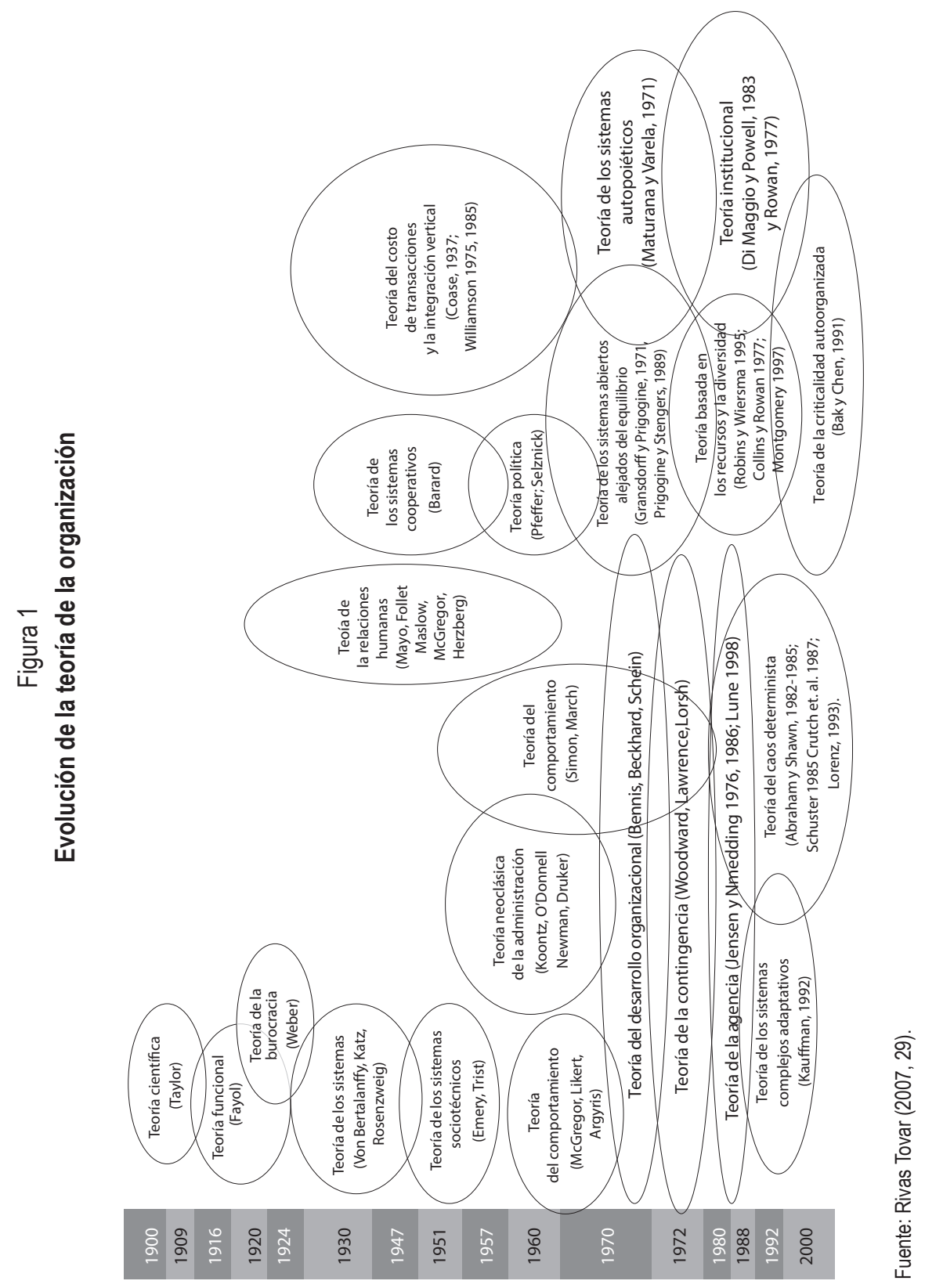


y gestión. Hay en esa obra tres aspectos centrales: el reconocimiento de la gestión como disciplina conceptual, la necesidad de que los ejecutivos desarrollen una visión integral y la formulación de un objetivo corporativo.

\section{Aprendizaje organizacional}

En vista de que las organizaciones aprenden a través de individuos que actúan como agentes, "se facilitan las actividades de aprendizaje de los individuos, a su vez, o son inhibidas por un sistema ecológico de factores que pueden llamarse sistema de aprendizaje organizacional" (Argyris 1977, 4). En este orden de ideas, McGill, Slocum y Lei (1992) definen el aprendizaje organizacional como la habilidad de una organización de ganar visión y comprensión a partir de experiencias a través de la experimentación, observación y análisis.

Senge (1993) señala que el aprendizaje adaptativo es aquel que se produce dentro de un marco de referencia establecido, sin cuestionarse el porqué del marco ni su idoneidad. Este tipo de aprendizaje es la base para las mejoras de tipo operativo, pero difícilmente busca nuevas formas de definir las claves del negocio.

\section{Desarrollo del modelo de gestión}

En coherencia con lo anteriormente señalado, los mejores modelos de organización y gestión mediática son aquellos que aciertan en su adecuado posicionamiento estratégico para cada uno de los eslabones de la cadena de valor. No se trata de entender la convergencia como un proceso de integración y concentración de recursos para optimizar costes aprovechando sinergias sino más bien como una oportunidad para reestructurar y rediseñar las cadenas de valor.

El modelo de organización en red (keiretsu en japonés) nace en Japón en la década de los ochenta, alrededor de la industria del automóvil y el aprovechamiento de las ventajas competitivas de la subcontratación. La jerarquía integrada es sustituida por redes flexibles de organizaciones unidas por acuerdos de colaboración. Acuerdos que pueden ser más o menos estables en función de las características sectoriales. En la industria del cine u otros 
sectores creativos, por ejemplo, esos acuerdos pueden ser de colaboración y competencia al mismo tiempo a través de conglomerados sectoriales. La organización reticular es más flexible, descentralizada, ligera, cooperativa y adaptable a los cambios, la creatividad y la innovación. Algunos autores sostienen que todas las organizaciones deben evolucionar en esa dirección para adaptarse mejor a la sociedad del conocimiento. Sin embargo, hay quien matiza también que "existen evidencias de que la organización cooperativa y flexible de la producción no está necesariamente relacionada con resultados superiores" (Villar-López y Camisón-Zornoza 2009).

Para implementar un modelo asociativo existen diferentes condiciones con las cuales se debe contar: confianza, precisión de objetivos y metas, compromiso, definición de roles, liderazgo y contrato integral.

\section{Fases del proceso de formación de una asociación}

Távara y Monja (2017) mencionan las diferentes etapas para las fases de proceso de formación que se deben tomar en cuenta:

- Identificación del promotor: identificar cuál es tarea para promover y qué interés tiene como asociación y demostrar el proyecto que es realista.

- Identificación de los miembros: se debería comenzar reuniendo a personas interesadas en el modelo asociativo con el fin de equiparar a productores adecuados para la asociación.

- Realización de un estudio de interés: una vez ya formada la asociación con el personal escogido se debe realizar un estudio de interés en cada uno de ellos.

- Designación de representantes: la organización escogerá una persona que se encargará de representarlos en las reuniones pertinentes.

- Organización de reuniones entre los miembros: después del primer encuentro se debe realizar reuniones para proponer mejoras en el modelo asociativo y las medidas que se están tomando deben ser más concretas con el fin de tener un objetivo claro y conciso.

- Realización de un estudio de viabilidad y redacción de un plan de actividades: una vez realizada la propuesta es necesario establecer un plan 
de actividades en el cual debe constar las necesidades y prioridades de las empresas, así como también su respectivo estudio de viabilidad.

- Constitución oficial del modelo asociativo: la formación del modelo asociativo se dará después de un lapso, la formación legal está basada en las reuniones, estudios de viabilidad y el plan de actividades.

- Plan de seguimiento: se basa en constatar el correcto funcionamiento de la empresa; se lo realizará por medio de continuas reuniones, sean individuales o colectivas, en las cuales se analizarán las estrategias a emplear.

\section{Materiales y métodos}

El artículo adopta la investigación teórica, documental, fundamentada en la literatura relacionada con la administración; descriptiva, ya que se basa en el estudio de las situaciones que ocurren en condiciones naturales, más que aquellas que se basan en situaciones experimentales.

Se fundamenta en el análisis de las variables de asociatividad y desarrollo empresarial que se dimensionan en la tabla 2, utilizando la estructura del FODA con matrices de ataque y defensa que a su vez generan los objetivos estratégicos.

- Matriz de ataque: se genera a partir de la relación de los factores externos de la empresa (fortalezas / oportunidades), utilizando una tabla de doble entrada y relacionándoles cada uno con una ponderación definida de la siguiente manera: $(5=$ alta, $3=$ media, $1=$ baja $)$.

Una vez terminada la relación, se tomaron los valores de las interacciones para formar la matriz de objetivos estratégicos.

- Matriz de defensa: se genera a partir de la relación de los factores internos de la empresa (debilidades / amenazas), utilizando una tabla de doble entrada y relacionándoles cada uno con una ponderación definida de la siguiente manera: $(5=$ alta, $3=$ media, $1=$ baja $)$.

Una vez terminada la relación, se tomaron los valores de las interacciones para formar la matriz de objetivos estratégicos.

Los objetivos estratégicos de la empresa se generan de las tablas de ataque y defensa, tomando en cuenta las dos ponderaciones más altas de cada una. 
Tabla 2

\section{Operacionalización de variables}

\begin{tabular}{|c|c|c|c|}
\hline Variables & Dimensión conceptual & Subvariables & Indicadores \\
\hline Asociatividad & $\begin{array}{l}\text { La asociatividad es una estrategia de co- } \\
\text { operación entre las pequeñas empresas } \\
\text { las cuales quieren un proceso de expan- } \\
\text { sión en conjunto en busca de un objetivo } \\
\text { común. }\end{array}$ & $\begin{array}{l}\text { Capacidades. } \\
\text { Recursos. } \\
\text { Servicios. }\end{array}$ & $\begin{array}{l}\text { Nivel de } \\
\text { confianza. } \\
\text { Servicios } \\
\text { básicos. } \\
\text { Bienes y capital } \\
\text { para utilizar. } \\
\text { Número de } \\
\text { miembros de la } \\
\text { asociación. }\end{array}$ \\
\hline $\begin{array}{l}\text { Desarrollo } \\
\text { empresarial }\end{array}$ & $\begin{array}{l}\text { Es la ejecución de las funciones admi- } \\
\text { nistrativas logrando ser eficiente en el } \\
\text { desarrollo empresarial. Es un proceso } \\
\text { en el cual intervienen diferentes varia- } \\
\text { bles tales como sociales, psicológicas y } \\
\text { económicas, que buscan tener una vida } \\
\text { empresarial exitosa. }\end{array}$ & $\begin{array}{l}\text { Panificación. } \\
\text { Organización. } \\
\text { Control. }\end{array}$ & $\begin{array}{l}\text { Gestión } \\
\text { administrativa. } \\
\text { Infraestructura. }\end{array}$ \\
\hline
\end{tabular}

Elaboración propia.

La identificación de las variables nos permite dimensionar la estructura del modelo de gestión, generando una estructura que nos permita conseguir el objetivo de la empresa.

\section{Resultados}

Se realizó un levantamiento del análisis de la situación actual mediante una herramienta FODA de la empresa "Don Jorge", seguida de una matriz de ataque y defensa según se detalla en la tabla 4 y 5 . 
Tabla 3

\section{Análisis FODA}

\begin{tabular}{|c|c|}
\hline Fortalezas & Oportunidades \\
\hline $\begin{array}{l}\text { F1. Terreno propio para la obtención de } \\
\text { materia prima. } \\
\text { F2. Disponibilidad de la materia prima. } \\
\text { F3. Compromiso familiar. } \\
\text { F4. Disponibilidad de mano de obra. }\end{array}$ & $\begin{array}{l}\text { O1: Clima favorable para el cultivo. } \\
\text { O2: Tendencia de consumo de productos naturales. } \\
\text { O3: Mercado de infusiones naturales amazónicas. } \\
\text { O4: Promoción del producto en ferias. }\end{array}$ \\
\hline Debilidades & Amenazas \\
\hline $\begin{array}{l}\text { D1: Sistema de riego inexistente. } \\
\text { D2: Manejo técnico. } \\
\text { D3: Transporte adecuado de materias } \\
\text { primas a la planta de proceso. } \\
\text { D3: Organización del trabajo. }\end{array}$ & $\begin{array}{l}\text { A1: Precios de mercado. } \\
\text { A2: Crisis económica mundial. } \\
\text { A3: Presencia de plagas. } \\
\text { A4: Clima. }\end{array}$ \\
\hline
\end{tabular}

Elaboración propia.

Tabla 4

Matriz de ataque

\begin{tabular}{|l|c|c|c|c|c|}
\hline \multicolumn{1}{|c|}{ Fortalezas } & $\begin{array}{c}\text { Terreno propio } \\
\text { para la obtención } \\
\text { de materia prima }\end{array}$ & $\begin{array}{c}\text { Utilización } \\
\text { de producto } \\
\text { natural }\end{array}$ & $\begin{array}{c}\text { Alto nivel de } \\
\text { compromiso en la } \\
\text { empresa familiar }\end{array}$ & $\begin{array}{c}\text { Disponibilidad } \\
\text { de mano de } \\
\text { obra }\end{array}$ & Total \\
\hline $\begin{array}{l}\text { Clima favorable } \\
\text { para el cultivo }\end{array}$ & 3 & 3 & 1 & 1 & 8 \\
\hline $\begin{array}{l}\text { Tendencia de } \\
\text { consumo a pro- } \\
\text { ductos naturales }\end{array}$ & 5 & 5 & 1 & 1 & 12 \\
\hline $\begin{array}{l}\text { Apertura en el } \\
\text { mercado }\end{array}$ & 1 & 3 & 4 & 1 & 9 \\
\hline $\begin{array}{l}\text { Existencia de } \\
\text { ferias agroin- } \\
\text { dustriales }\end{array}$ & 1 & 5 & 3 & 1 & 9 \\
\hline Total & 10 & 16 & 9 & 4 & \\
\hline
\end{tabular}

Elaboración propia. 
Tabla 5

Matriz de defensa

\begin{tabular}{|l|c|c|c|c|c|}
\hline \multicolumn{1}{|c|}{ Amenazas } & $\begin{array}{c}\text { Precios } \\
\text { de mercado }\end{array}$ & $\begin{array}{c}\text { Crisis } \\
\text { económica } \\
\text { mundial }\end{array}$ & $\begin{array}{c}\text { Presencia } \\
\text { de plagas }\end{array}$ & $\begin{array}{c}\text { Presencia } \\
\text { de lluvia } \\
\text { constante }\end{array}$ & Total \\
\hline $\begin{array}{l}\text { Sistema de riego } \\
\text { inexistente }\end{array}$ & 1 & 4 & 1 & 3 & 9 \\
\hline $\begin{array}{l}\text { Falta de manejo } \\
\text { técnico }\end{array}$ & 3 & 3 & 5 & 3 & 13 \\
\hline $\begin{array}{l}\text { Transporte } \\
\text { deficiente }\end{array}$ & 1 & 1 & 1 & 3 & 6 \\
\hline $\begin{array}{l}\text { Falta de trabajo } \\
\text { organizado }\end{array}$ & 1 & 3 & 1 & 1 & 6 \\
\hline Total & 6 & 11 & 8 & 10 & \\
\hline
\end{tabular}

Elaboración propia.

\section{Propuesta del modelo de gestión asociativo}

La propuesta del proyecto se basa en establecer un modelo de gestión asociativo en la empresa agroindustrial "Don Jorge" que parte generando la marca y eslogan según se detalla en la tabla 6.

\section{Marca y eslogan}

Tanto la marca como el eslogan se generan por el creador de empresa de té verde, basado en un círculo familiar de acuerdo con lo establecido en la tabla 6. 
Análisis de caso del modelo de gestión asociativo de la empresa agroindustrial "Don Jorge"

Tabla 6

\section{Marca y eslogan}

\begin{tabular}{|l|l|}
\hline \multicolumn{1}{|c|}{ Marca } & \multicolumn{1}{|c|}{ Eslogan } \\
\hline $\begin{array}{l}\text { La marca proviene de una historia familiar, en } \\
\text { honor al nombre del fundador de la empresa, } \\
\text { señor Jorge Aníbal Jiménez. }\end{array}$ & $\begin{array}{l}\text { Se basa en el consumo de materias primas que } \\
\text { brindan alternativas naturales a los consumido- } \\
\text { res, ofertando un sabor exótico que invita a los } \\
\text { consumidores a elegir y a vivir de forma más } \\
\text { saludable. }\end{array}$ \\
Té Verde 100\% Natural \\
\hline
\end{tabular}

Elaboración propia.

\section{Objetivos estratégicos}

La tabla 7 resulta de las matrices de ataque y defensa, en relación con su ponderación se definen los parámetros que serán parte del objetivo.

Tabla 7

Matriz de objetivos estratégicos

\begin{tabular}{|l|l|}
\hline Externos & $\begin{array}{l}\text { Fomentar el consumo de productos naturales. } \\
\text { Identificar los posibles mercados de consumo. }\end{array}$ \\
\hline Internos & $\begin{array}{l}\text { Capacitar al personal sobre el manejo de los cultivos. } \\
\text { Implementar un sistema de riego que permita optimizar los recursos hídricos. }\end{array}$ \\
\hline
\end{tabular}

Elaboración propia. 


\section{Prácticas principales del modelo de gestión de la empresa "Don Jorge"}

Se toma en cuenta el concepto de Osterwalder, Pigneur y Tuccini (2005), que define que un modelo de negocio es una herramienta conceptual que contiene un conjunto de elementos y sus relaciones y que nos permite expresar la lógica de negocio de una empresa específica. La estructura del modelo de gestión de la empresa se enfoca en 4 aspectos fundamentales: estrategia, ejecución, estructura y cultura, que nos permiten ejecutar las estrategias definidas.

Figura 2

Modelo de gestión

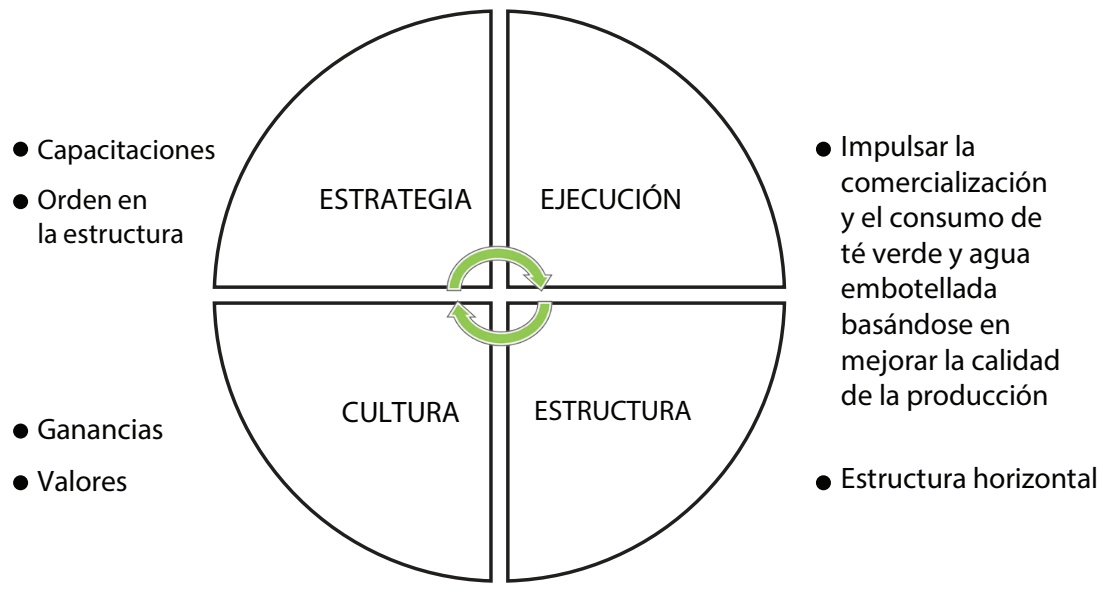

Elaboración propia. 


\section{Estructura del modelo de gestión de "Don Jorge"}

La empresa "Don Jorge" contará con un modelo de gestión empresarial que ayudará a la toma decisiones. El modelo de gestión se basa en dos pilares fundamentales como es la asociatividad y el desarrollo empresarial con parámetros que mejoran su eficiencia, tales como: promoción, confianza, plan estratégico y la autogestión, según se define en la figura 3.

Figura 3

Estructura del modelo de gestión

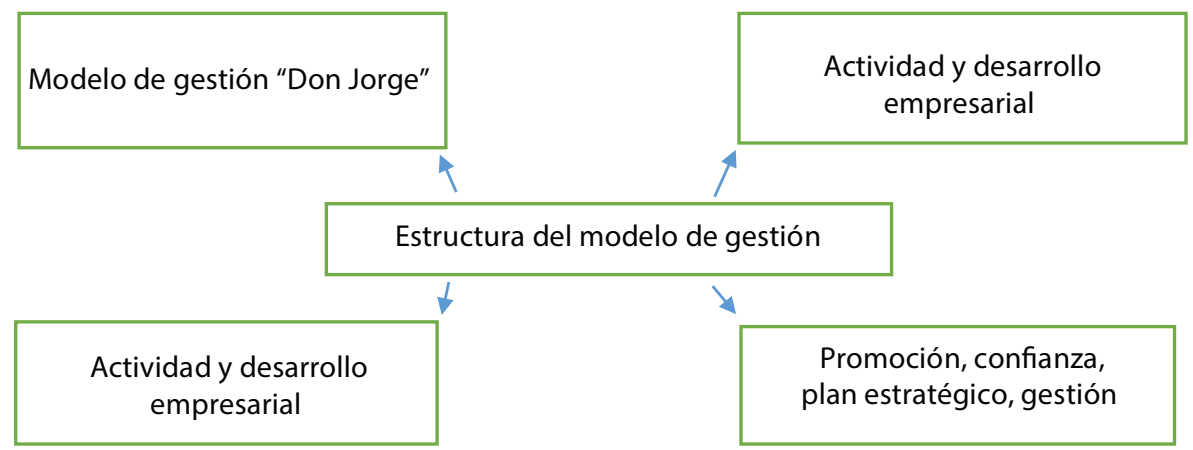

Elaboración propia.

\section{Elementos que constituyen el modelo}

- Asociatividad: es considerado como un trabajo colectivo social. La unión de diferentes organizaciones con un propósito que no pueden cumplir individualmente con desarrollo de principios y valores como confianza y compromiso.

- Desarrollo empresarial: es la ejecución de las funciones administrativas logrando ser eficiente en el desarrollo empresarial. En este proceso intervienen diferentes variables tales como sociales, psicológicas y económicas, que buscan tener una vida empresarial exitosa (Varela y Bedoya 2006). 
Tabla 8

\section{Estructura de un modelo asociativo}

\begin{tabular}{|c|c|c|}
\hline Fases & Concepto & Pasos \\
\hline Promoción & $\begin{array}{l}\text { Son los beneficios que tiene la organiza- } \\
\text { ción entre los socios. }\end{array}$ & $\begin{array}{l}\text { 1. Promoción de ideas. } \\
2 \text {. Identificación de los diferentes } \\
\text { socios. } \\
\text { 3. Evaluar los principales facto- } \\
\text { res que están ligados a la aso- } \\
\text { ciatividad. } \\
\text { 4.Selección de personal para el } \\
\text { trabajo. }\end{array}$ \\
\hline Confianza & $\begin{array}{l}\text { Es un factor importante para la realiza- } \\
\text { ción de actividades dentro de la organi- } \\
\text { zación por parte de los socios. }\end{array}$ & $\begin{array}{l}\text { 1. Reuniones entre los socios. } \\
\text { 2. Evaluación de un diagnóstico } \\
\text { de la empresa. } \\
\text { 3. Instauración de un contrato } \\
\text { con términos de negociación den- } \\
\text { tro de la asociación. }\end{array}$ \\
\hline Plan estratégico & $\begin{array}{l}\text { Se enmarca en un proceso de coope- } \\
\text { ración asociativa esto depende de los } \\
\text { objetivos la constitución jurídica de cor- } \\
\text { poración interna. }\end{array}$ & $\begin{array}{l}\text { 1. Tomar en cuenta los ajustes } \\
\text { empresariales. } \\
\text { 2. Desarrollar las bases el diseño } \\
\text { del proyecto. } \\
\text { 3. Realizar o iniciar las actividades. }\end{array}$ \\
\hline Autogestión & $\begin{array}{l}\text { Realizar y consolidar las mismas bases } \\
\text { de autorrealización. }\end{array}$ & $\begin{array}{l}\text { 1. Designar un representante. } \\
\text { 2. Iniciar las bases del proyecto. } \\
\text { 3. Seguimiento de las actividades. }\end{array}$ \\
\hline
\end{tabular}

Elaboración propia.

La estructura del modelo asociativo consta de cinco fases importantes, según se muestra en la tabla 8.

Enríquez y Montenegro (2020) plantean en su modelo de gestión asociativo a nivel rural de un centro de acopio de fréjol en la comuna Las Rocas de la provincia de Chimborazo, la importancia de la asociatividad y el mercado ya que con base en estas se logra la planeación y definir los objetivos de la organización, establecer estrategias para lograr dichos objetivos y desarrollar planes para integrar y coordinar actividades de trabajo. Reinel (2005) sugiere que se organicen en modelos asociativos para participar en el mercado inter- 
nacional, justo en el momento en que la crisis económica mundial continúa siendo un tema que suscita preocupación, las relaciones comerciales con los principales socios comerciales. Si bien se promueve la asociatividad entre empresas, también es pertinente que, para propiciar este tipo de encadenamientos, previamente se hayan armonizado las relaciones entre las instituciones reguladoras y de promoción, públicas y privadas. De esta manera, se empezaría por aplicar en casa de estos organismos una filosofía de cooperación y ayuda, que coadyuve al éxito de los encadenamientos de exportación. La idea es la de apuntar a una cadena integral en la que participen las empresas y los entes estatales propios del contexto, puesto que el desarrollo económico requiere coordinación entre los distintos sectores, incluyendo el papel del gobierno (Stiglitz y Charlton 2007).

\section{Conclusiones}

El levantamiento de información del modelo de gestión asociativo se lo realizó con ayuda de fuentes bibliográficas confiables las cuales nos conducen a concluir que el modelo de gestión asociativo es considerado una herramienta para el mejoramiento de una estructura empresarial estratégica, que ayudará al cumplimiento de los objetivos propuestos por la empresa "Don Jorge" y lograr tener éxito a largo plazo.

El té ha causado un impacto positivo frente a la sociedad ya que es una bebida muy consumida a nivel mundial y su principal razón de beberla es por salud.

Al aplicar el modelo asociativo en la empresa agroindustrial "Don Jorge", ofrece una mayor organización sobre la estructura interna y la cadena productiva, ayudando a hacer más eficaz al momento de la toma de decisiones. El modelo se basa en dos pilares fundamentales como es la asociatividad y el desarrollo empresarial con estrategias claves para el crecimiento de la organización y el cumplimiento de objetivos comunes, así como también para desarrollar las actividades de la empresa establecidas. 


\section{Referencias}

Alegre, Luis, Carmen Berné Manero y Carmen Galve Górriz. 2008. "La empresa". En Fundamentos de economía de la empresa: perspectiva funcional, editado por Luis Alegre, 9-24. Barcelona: Ariel Economía.

Argyris, Chris. 1977. El individuo dentro de la organización. Barcelona: Herder.

Banco Internacional de Reconstrucción y Fomento (BIRF). 2009. Una nueva geografía económica. Informe sobre el desarrollo mundial. Accedido diciembre de 2020. www.iadb. org/es/acerca-del-bid/perspectiva-general.

Belausteguigoitia, Imanol. 2008. Empresas familiares: su dinámica, equilibrio y consolidación. Ciudad de México: McGraw-Hill.

Caldas, María, Ricardo Carrión y José Antonio Heras. 2009. Empresa e iniciativa emprendedora. Madrid: Editex.

Campiña Domínguez, Gema, y María Fernández. 2010. La empresa y su entorno. Madrid: Editex.

Contreras Soto, Ricardo. 2010. "Reflexiones en torno a la construcción científica en el campo de los estudios de las organizaciones, gestión y administración en México". Observatorio de la Economía Latinoamericana 127: 20-56. https://bit.ly/3vOKie8.

Corporación Andina de Fomento (CAF). 2005. América Latina en el comercio global. Ganando mercados. Caracas: CAF.

Drucker, Peter. 1954. The Practice Management. Nueva York: Harper \& Row.

Elizondo, Ricardo. 2015. La economía social y solidaria en Ecuador. Madrid: Agencia Vasca para el Desarrollo.

Enríquez, Miguel, y Karina Montenegro. 2020. “Análisis de caso del modelo de gestión asociativo a nivel rural en acopio de fréjol, en la comuna Las Rocas cantón Alausí, provincia de Chimborazo". Revista Economía y Negocios UTE 11 (1): 34-46. https://bit.ly/3n0Lmuz.

Enríquez, Miguel, y Manuel Pérez. 2020. "Perspectiva de consumo y marketing mix para una infusión de ortiga con naranja en Pastaza”. Revista ECA Sinergia, 11 (2): 34-46.

Fernández, Enrique de Miguel. 2005. Introducción a la gestión empresarial. Valencia: Universidad Politécnica de Valencia.

Fundación Observatorio PyME. "Informe 2015-2016. Evolución reciente, situación actual y desafíos para 2017". Accedido diciembre de 2020. https://bit.ly/3zRxc2P.

Gil Estallo, María de los Ángeles. 2007. La organización de empresas: hacia un modelo de futuro. Madrid: ESIC.

McGill, Michael, David Lei y John Slocum, 1992. "Management Practices in Learning Organizations”. Organizational Dynamics 21: 5-17. 
Análisis de caso del modelo de gestión asociativo de la empresa agroindustrial "Don Jorge"

Osterwalder, Alexander, Yves Pigneur y Christopher Tucci. 2005. “Clarifying Business Models: Origins, Present, and Future of the Concept. Communications of the Association for Information Systems" AIS 15: 751-775. https://doi.org/10.17705/1CAIS.01601.

Porter, Michael. 1997. Ventaja competitiva. Creación y sostenimiento de un desempeño superior. Ciudad de México: Diana.

Reinel, Pulecio Jorge. 2005. "La estrategia Uribe de negociación del TLC”. Revista Colombia Internacional 61: 34-49.

Rivas Tovar, Luis Arturo. 2007. "Mapa de las teorías de la organización: una orientación para empresas". Gaceta Ideas 23: 35-67.

Senge, Peter. 1993. La quinta disciplina. El arte y la práctica de la organización abierta al aprendizaje. Buenos Aires: Granica.

Stiglitz, Joseph, y Andrew Charlton. 2007. Comercio justo para todos. Cómo el comercio puede promover el desarrollo. Bogotá: Taurus.

Torres, Juan, Sebastián. 2016. "Modelo de gestión asociativo para los productores de yuca de la parroquia San Jacinto". Tesis de posgrado, Universidad Andina Simon Bolívar, Sede Ecuador, Quito.

Torres Valdivieso, Sergio. 2001. “Cambio organizacional y evolución tecnológica: la máquina-herramienta en el país vasco". Cuadernos de Administración 14 (23): 7-23.

Varela, Rodrigo, y Arturo Bedoya. 2006. "Modelo conceptual de desarrollo empresarial basado en competencias". Estudios Gerenciales 100: 12-47. https://bit.ly/3wSIPEH.

Vargas, German, y Gloria Calvo. 1987. "Seis modelos alternativos de investigación documental para el desarrollo de la práctica universitaria en educación”. Revista Educación Superior y Desarrollo 5 (3): 7-37. 